\title{
Modeling the functional connectivity in embodied in vitro neuronal network
}

\author{
Antonio Novellino*1, Michela Chiappalone ${ }^{2,3}$ and Sergio Martinoia ${ }^{2,3}$
}

\author{
Address: ${ }^{1}$ Institute for Health Consumer and Protection, Joint Research Centre, Ispra (VA), 21020, Italy, ${ }^{2}$ Department of Biophysical and Electronic \\ Engineering, University of Genova, Genova, 16145, Italy and ${ }^{3}$ Department of Neuroscience and Brain Technologies, Italian Institute of \\ Technology, Genova, 16163, Italy \\ Email: Antonio Novellino* - antonio.novellino@jrc.it \\ * Corresponding author
}

from Eighteenth Annual Computational Neuroscience Meeting: CNS*2009

Berlin, Germany. 18-23 July 2009

Published: 13 July 2009

BMC Neuroscience 2009, I0(SuppI I):PI 46 doi:I0.II86/I47I-2202-I0-SI-PI 46

This abstract is available from: http://www.biomedcentral.com/I47I-2202/I0/SI/PI46

(c) 2009 Novellino et al; licensee BioMed Central Ltd.

\section{Introduction}

We developed a hybrid neuro-robotic bi-directional interface connecting in-vitro neuronal networks to a small mobile robot to investigate neural processes and functional modification that underlie sensorimotor learning in the nervous system $[1,2]$. We describe the investigation of the effect of the stimulus-induced distributed plasticity and functional connectivity on the information processing capabilities of the neuronal network. The results evidence a modification at functional connectivity level that can be described in terms of a Hebbian learning rule.

\section{Results}

We can model our neuronal network with two input nodes (i.e. feedback stimulation sites) and two output nodes (recoding sites) and we can map functional connectivity among these I/O pathways: the post stimulus time histogram (PSTH) represent the firing probability (of the output node) after a given stimulus (from the input node). Hebb suggested that reverberating activity in assemblies of neurons carries a memory trace that become permanent and it is manifested as changes in synaptic weights between the neurons that persistently take part to firing [3]. A very simple formulaic description of Hebbian learning is:

$$
\omega_{i j}=\frac{1}{n} \sum_{k=1}^{p} x_{i}^{k} x_{j}^{k}
$$

Where $\omega_{i j}$ is the weight of connection from node $j$ to node $i, n$ is the dimension of the input pattern, $p$ is the number of training patterns and $x_{i}^{k}$ is the $k$-th input for node $i$. In our model we have two stimulation sites and two recording sites:

$$
\left[\begin{array}{cc}
\omega_{L L}=\frac{1}{n} \sum_{k=1}^{p} x_{L}^{k} x_{L}^{k} & \omega_{R L}=\frac{1}{n} \sum_{k=1}^{p} x_{R}^{k} x_{L}^{k} \\
\omega_{L R}=\frac{1}{n} \sum_{k=1}^{p} x_{L}^{k} x_{R}^{k} & \omega_{R R}=\frac{1}{n} \sum_{k=1}^{p} x_{R}^{k} x_{R}^{k}
\end{array}\right]
$$

If we apply it to a limited time window (hundreds of ms) we have applied the formula to compute the Post Stimulus Time Histogram. Thus the description of the functional connectivity in terms of the area of the PSTH can be considered a descriptor to evidence the induced modulations in terms of modulation of hebbian weights and the results from the closed loop session (Figure 1).

The hypothetical configuration for the obstacle avoidance achievement requires the cross-connection weights to be weaker than the direct ones. The achieve modulation is close to the theoretical achievement thus confirming the possibility to describe the embodied neuro-robotic paradigm in terms of Hebbian rules. 

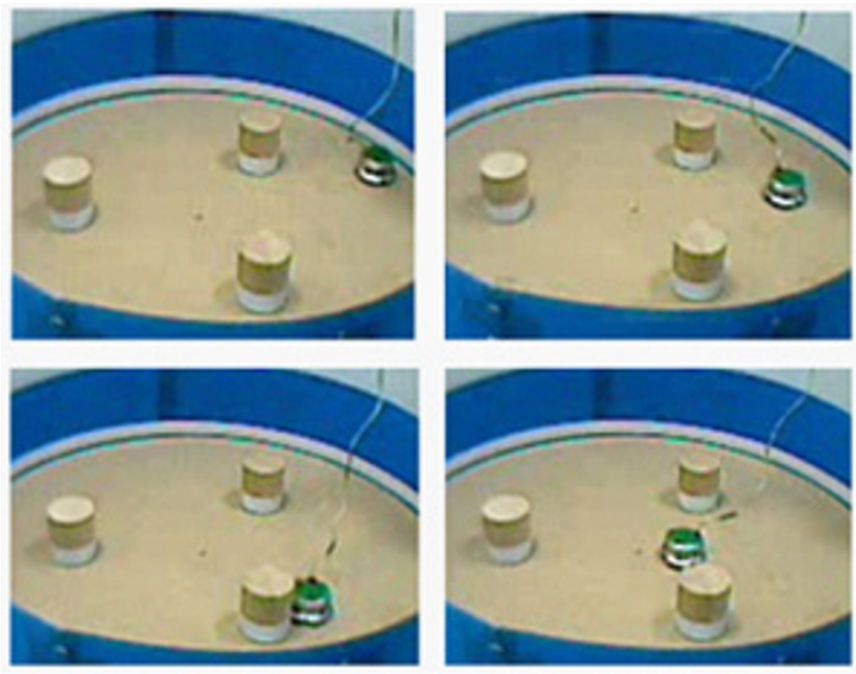
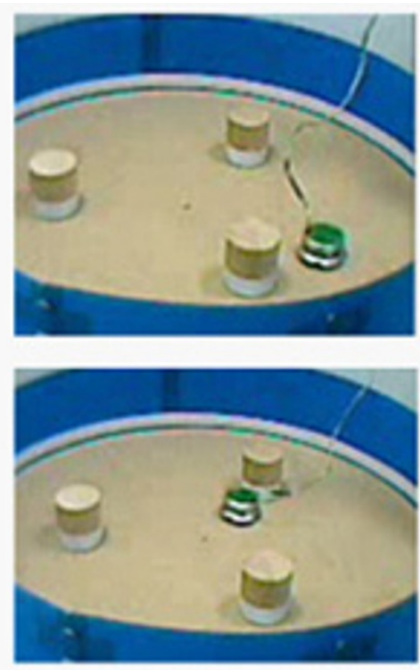
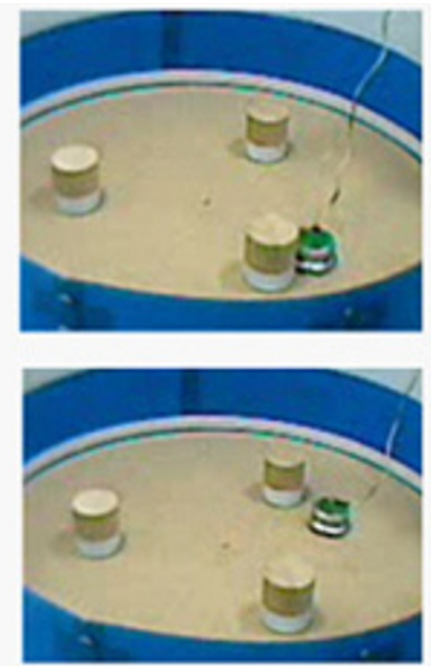

\section{Figure I}

The robot, driven by the network's activity, wandering inside the arena during an experimental session (frame frequency $0.5 \mathrm{~Hz}$ ).

\section{References}

I. Novellino A, D'Angelo P, Cozzi L, Chiappalone M, Sanguineti V, Martinoia $S$ : Connecting neurons to a mobile robot: an in vitro bidirectional neural interface. Comput Intell Neurosci 2007:I-I3. doi: $10.1155 / 2007 / 12725$.

2. Cozzi L, D'Angelo P, Sanguineti V: Encoding of time-varying stimuli in populations of cultured neurons. Biol Cybern 2006, 94:335-349. doi: 10.1007/s00422-006-005I-2

3. Hebb DO: Organization of Behavior John Wiley \& Sons, New York; 1949.

Publish with Biomed Central and every scientist can read your work free of charge

"BioMed Central will be the most significant development for disseminating the results of biomedical research in our lifetime. "

Sir Paul Nurse, Cancer Research UK

Your research papers will be:

- available free of charge to the entire biomedical community

- peer reviewed and published immediately upon acceptance

- cited in PubMed and archived on PubMed Central

- yours - you keep the copyright
BioMedcentral 